J. Phys. IV France 127 (2005) 77-85

(C) EDP Sciences, Les Ulis

DOI: $10.1051 /$ jp4:2005127012

\title{
Un spectromètre par transformation de Fourier sans lame séparatrice, pour le VUV-XUV. Principes, réalisation, et premiers résultats
}

\author{
D. Joyeux ${ }^{1}$, N. de Oliveira ${ }^{2}$, D. Phalippou ${ }^{1}$, J.C. Rodier ${ }^{1}$, M. Vervloet ${ }^{3}$, \\ F. Polack ${ }^{2}$ et L. Nahon ${ }^{2}$
}

${ }^{1}$ Laboratoire Charles Fabry de I'Institut d'Optique, CNRS, Bât. 503, Université Paris-Sud, 91405 Orsay Cedex, France

2 Synchrotron Soleil, Orme des Merisiers, St. Aubin, BP. 48, 91192 Gif-sur-Yvette Cedex,

France

${ }^{3}$ Laboratoire de Photophysique Moléculaire du CNRS, Bât. 350, Université Paris-Sud, 91405 Orsay Cedex, France

\begin{abstract}
Résumé. La spectrométrie par transformation de Fourier est une technique maintenant bien maitrisée, qui permet d'obtenir des spectres à la fois exacts et à très haute résolution, de l'infra-rouge lointain à l'ultra-violet, jusque vers 140-120 nm. En dessous, en particulier de 140 à $20 \mathrm{~nm}$ environ, la technique est complètement absente, essentiellement en raison de la quasi impossibilité de fabriquer des lames séparatrices ayant les qualités requises. Or, dans ce domaine, la physique atomique et moléculaire est demandeuse d'une précision et d'une résolution que n'apportent que partiellement les spectromètres à réseaux utilisés. Nous présentons les principes et la réalisation d'un interféromètre à balayage dédié à la spectrométrie TF jusque $\lambda=58 \mathrm{~nm}$, avec une résolution au moins égale à 400000 à $80 \mathrm{~nm}$. Le problème de la séparatrice est résolu radicalement par l'utilisation d'un interféromètre à division de front d'onde. La précision du balayage en différence de marche est obtenue grâce à un système original de contrôle et de mesure du déplacement du réflecteur mobile. Nous présentons les premières mesures réalisées dans l'air, vers $200 \mathrm{~nm}$ (bande d'absorption de $\mathrm{O}_{2}$, source synchrotron), avec les paramètres de balayage du système nominal (500 000 échantillons espacés de $30 \mathrm{~nm}$ env., TF en cosinus).
\end{abstract}

\section{INTRODUCTION}

La spectrométrie par transformation de Fourier (STF) est une technique maintenant bien établie, et utilisée dans un très vaste domaine spectral, allant de l'UV proche $(\lambda \geq 140 \mathrm{~nm})$ à l'IR très lointain. Elle résulte des travaux pionniers de Michelson (1891-1892), puis Rubens et Wood (1911), mais ne fut véritablement développée qu'à partir des années 1950 et surtout 1960. Elle repose sur la relation existant entre la luminance spectrale d'une source (fonction du nombre d'onde $1 / \lambda$ ) et l'interférogramme qu'on obtient en éclairant avec cette source un interféromètre à deux ondes balayé en différence de marche. Cette relation est une simple transformation de Fourier sur le couple différence de marche - nombre d'onde. Les deux qualités principales intrinsèques à la STF sont: une résolution spectrale potentiellement très élevée $\left(10^{6}\right)$, et une bonne exactitude du spectre en large bande (position, forme et amplitude des éléments spectraux), ce qui n'est pas toujours le cas avec les meilleurs spectromètres à réseaux. Or, la STF est inconnue en dessous de $140 \mathrm{~nm}$, bien que ces qualités subsistent potentiellement aux longueurs d'ondes plus courtes. Cet état de fait résulte principalement d'un blocage technologique: la difficulté, voire l'impossibilité de réaliser les lames séparatrices transmission/réflexion, des interféromètres utilisés classiquement, Michelson ou Mach-Zehnder.

Cette difficulté, qui est liée à la très forte absorption des matériaux dans le domaine spectral EUV, se rencontre dès qu'on parle d'interférométrie à courte longueur d'onde. Même si, dans certains domaines spectraux, il est envisageable, et actuellement tenté, de réaliser des lames séparatrices de 
qualité interférométrique, il existe par ailleurs une solution radicale au problème. On peut utiliser des interféromètres qui par nature se passent de séparatrice, à savoir les interféromètres dits à division de front d'onde (ex: le bimiroir de Fresnel), par opposition aux interféromètres à division d'amplitude, type Michelson. Ayant déjà mis en œuvre ce type d'interféromètre à des longueurs d'ondes nanométriques [1], il nous a semblé raisonnable de l'appliquer au problème de la STF. Bien entendu, il reste à résoudre une autre difficulté (qui n'est pas un blocage), liée au fait que la précision des montages et des indispensables mesures de différence de marche (ddm) est toujours inversement proportionnelle à la longueur d'onde.

Dans ces conditions, nous nous sommes fixé comme but la réalisation d'un interféromètre à division de front d'onde balayé en ddm, pour la spectrométrie TF en large bande dans le domaine spectral 140-60 $\mathrm{nm}$, en raison de son intérêt pour la spectroscopie atomique et moléculaire. La résolution spectrale visée a été fixée à 500000 à $60 \mathrm{~nm}$. On notera que le meilleur système à réseau en fonctionnement sur anneau synchrotron donne $\mathrm{R}=200000$ à $80 \mathrm{~nm}$ [2].

\section{UN INTERFÉROMÈTRE BALAYÉ EN DIFFÉRENCE DE MARCHE DANS LE VUV-EUV}

L'un des montages prototypes des interféromètres sans séparatrice est le bimiroir de Fresnel représenté sur la figure 1. Dans ce montage, l'onde incidente éclaire les deux miroirs à cheval sur l'arête commune, et est donc divisée spatialement en deux demi-ondes. Pour obtenir un champ d'interférence utilisable, on incline l'un des miroirs par rapport à l'autre, de façon à obtenir à distance du bimiroir une zone de recouvrement, dans laquelle les franges peuvent être observées. On remarque que le nombre de franges observables et l'interfrange ne sont pas indépendants, et qu'en toute rigueur, il est impossible avec un tel système d'obtenir une teinte plate. D'autre part, l'angle d'incidence des faisceaux est un paramètre libre, qu'il est possible d'ajuster pour une réflectivité correcte. Ceci permet d'éviter les traitements multicouches, qui, outre le fait qu'ils ne sont pas toujours disponibles, rendraient l'instrument fortement chromatique. Au contraire, une seule couche bien choisie ( $\mathrm{Pt}$, ou mieux SiC) est faiblement chromatique, et permet de fonctionner sur toute la bande spectrale visée avec les mêmes réflecteurs. Enfin, la structure bimiroir se prête bien à une fabrication compacte, stable et relativement facile à aligner et à régler à la différence de marche nulle.

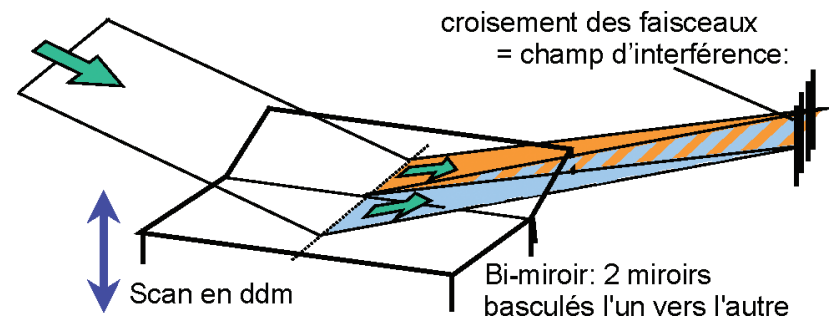

Figure 1. Montage prototype des interféromètres à division de front d'onde. Le balayage en différence de marche est en principe possible.

\subsection{Principe et objectifs}

En principe, le schéma de la figure 1 permet, en translatant l'un des miroirs perpendiculairement à lui même, de réaliser un balayage en différence de marche en tout point du champ d'interférence. Toutefois, l'interférogramme obtenu ne serait exact en toute rigueur qu'avec des faisceaux spatialement uniformes et larges, et de grande cohérence spatiale: en effet les rayons interférant en un point du champ proviennent de points sources variables lorsque la différence de marche varie. Avec des faisceaux réels, spécialement s'ils sont de cohérence spatiale limitée et peu uniformes, il est indispensable que les rayons interférant soient invariants pendant le balayage. Ceci est réalisé si l'on substitue à chaque miroir de la figure 1, un 
ensemble de deux miroirs formant un dièdre (miroir en toit), le balayage étant réalisé selon la direction bissectrice du dièdre mobile (fig. 2). Comme avec le bimiroir simple, l'un des dièdres doit être basculé vers l'autre pour assurer le croisement des faisceaux interférant. Dans cette configuration, l'angle d'incidence sur chaque miroir reste le paramètre libre qui fixe l'angle du dièdre. Son choix est en partie gouverné par la réflectivité qu'il est possible d'obtenir, c'est à dire par la gamme spectrale visée, à savoir $\lambda \geq 60 \mathrm{~nm}$ (cf. sect. 1). Dans ces conditions, nous avons choisi d'utiliser une incidence de 45 deg sur les miroirs, ce qui permet d'obtenir une réflectivité globale acceptable avec un traitement simple couche. Le principal intérêt de cette configuration est que, l'angle du dièdre étant $90 \mathrm{deg}$, le faisceau réfléchi par le dièdre mobile utilise toujours la même partie du réflecteur au cours du balayage: il est donc tout à fait insensible, au moins au premier ordre, à d'éventuelles erreurs de forme. On montre facilement que la relation entre le déplacement du dièdre mobile et la différence de marche qu'elle induit est: $d d m=2 \times$ déplacement. Il reste à préciser l'angle de basculement des dièdres, c'est à dire la structure du champ d'interférence, en vue d'une détection optimale de l'état d'interférence en fonction du balayage.

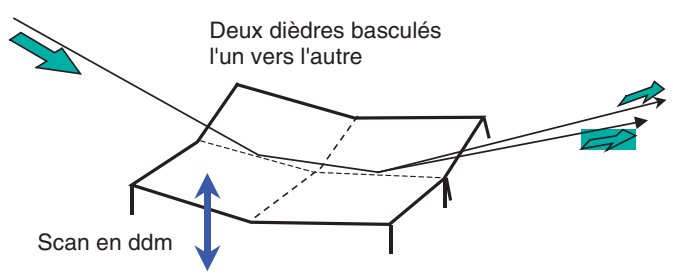

Figure 2. Montage en principe équivalent au montage de gauche. En remplaçant chaque miroir plan par un dièdre, et en translatant un dièdre parallèle à son plan bissecteur, les trajets hors dièdre sont invariants pendant la translation.

\subsection{Réglage de l'interférogramme}

Comme il est rappelé en introduction, la première étape de la STF consiste à mesurer l'état d'interférence en fonction de la différence de marche. Dans la mesure où le champ d'interférence n'est pas une teinte plate, la ddm varie perpendiculairement aux franges. Le détecteur doit donc être une fente parallèle aux franges, et plus petite qu'un interfrange, 1/4 à 1/3 de frange pour ne pas trop atténuer la modulation de l'interférogramme. L'éclairement moyen du plan d'interférence étant indépendant de l'angle de croisement, on voit qu'il y a avantage, du point de vue du flux moyen détecté et donc du rapport signal/bruit, à utiliser le plus grand interfrange possible. Or, comme on l'a signalé plus haut, les 2 paramètres définissant le champ d'interférence: largeur et interfrange, sont couplés à travers l'angle de croisement des faisceaux et la distance du plan d'observation aux réflecteurs. En supposant que l'interféromètre est éclairé par une fente source placée à $\mathrm{D}$ en amont de l'interféromètre, et que la détection est placée à $\mathrm{D}$ en aval (fig. 3), on a largeur $d u$ champ $=\gamma \mathrm{D}$ et interfrange $=2 \lambda / \gamma$ avec $\gamma=$ angle de croisement des faisceaux. (Ici, le champ est défini par l'ombre géométrique des bords communs des réflecteurs). On voit donc que l'interfrange augmente et le champ diminue lorsque $\gamma$ diminue. La situation optimale est donc obtenue lorsque le champ d'interférence contient une seule frange. Dans l'approximation géométrique, cette condition se réduit à $\gamma \mathrm{D}=2 \lambda / \gamma$, d'où $\gamma=\sqrt{2 \lambda / \mathrm{D}}$ et interfrange $=$ $\sqrt{2 \lambda \mathrm{D}}$. A titre d'exemple, avec $\lambda=60 \mathrm{~nm}, \mathrm{D}=800 \mathrm{~mm}$, on trouve $\gamma=0.39 \mathrm{mrad}$ et interfrange $=$ $310 \mu \mathrm{m}$.

En réalité, une analyse plus fine est nécessaire pour tenir compte de trois facteurs: 1) la diffraction de Fresnel par l'arête commune des réflecteurs est prépondérante dans la formation du champ d'interférence. En effet, la pseudo période de Fresnel dans le plan d'observation est elle même, dans les mêmes conditions, $\sqrt{2 \lambda \mathrm{D}}$. 2) L'optimisation repose sur le rapport s/b, le signal étant la modulation du flux détectée en fonction de la ddm, et le bruit étant donné de façon ultime par le bruit de photon, donc par le flux moyen détecté. 


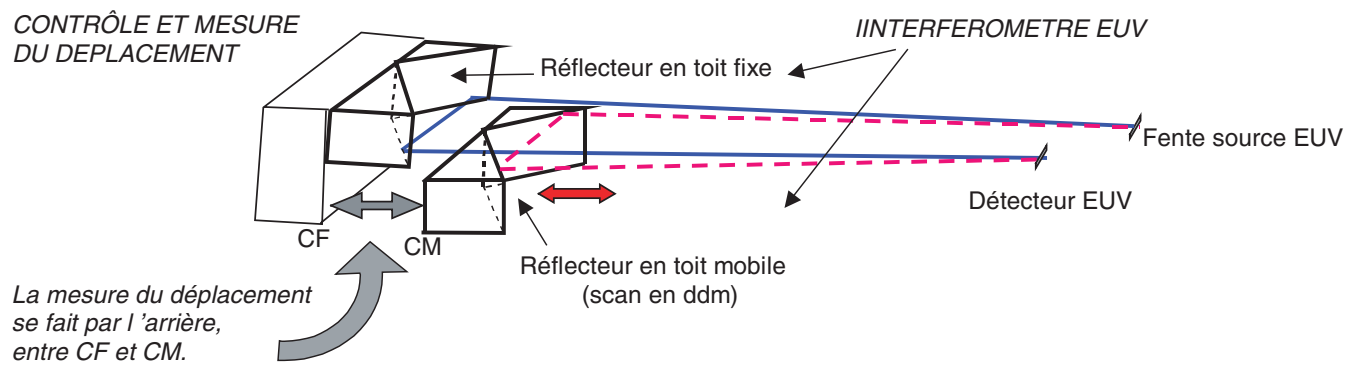

Figure 3. Le système est constitué de deux blocs optiques "solides", l'un affecté au bras fixe, l'autre au bras mobile. Chaque bloc est constitué d'un partie EUV (dièdre, regardant vers la source et le détecteur EUV), et d'un miroir plan placé derrière le dièdre. Le système de contrôle mesure en permanence la stabilité angulaire et la variation de position relative de ces deux miroirs plans.

Dans ces conditions, la largeur de la fente de détection est aussi un paramètre d'optimisation. De plus, selon les sources utilisées, il peut être nécessaire ou non, de tenir compte de la cohérence spatiale des ondes interférentes, elle même déterminée par la dimension d'une fente source convenablement placée. 3) Enfin, il est évidemment nécessaire de ménager une "garde" entre le réflecteur fixe et le réflecteur mobile. Cette garde peut être de l'ordre de 50 à $100 \mu \mathrm{m}$, c'est à dire pas beaucoup plus petite que le recouvrement des ondes. Ceci perturbe donc le calcul simple précédent, et conduit à augmenter le basculement $\gamma$. Au total, on trouve que la détection est optimale vers $60 \mathrm{~nm}$ avec $\gamma=0.9 \mathrm{mrad}$, d'où un interfrange de 130 $\mu \mathrm{m}$. La fente de détection ne doit donc pas être plus grande que $50 \mu \mathrm{m}$ (donnant une atténuation tolérable de 0.78 à $\lambda=60 \mathrm{~nm}, 0.87$ à $80 \mathrm{~nm})$.

La configuration géométrique de l'interféromètre EUV et du système de frange est ainsi complètement fixée. Il reste à déterminer ses spécifications dynamiques: amplitude et qualité du balayage en différence de marche, en vue d'obtenir des spectres "exacts", c'est à dire pour lesquels les erreurs sur la mesure de l'interférogramme ne se traduisent pas par des déformations spectrales intolérables. L'analyse de cette question étant classique, nous n'en donnerons pas les détails, qui peuvent par exemple être trouvés dans Chamberlain [3].

\subsection{Qualité du balayage en différence de marche}

Rappelons d'abord l'objectif visé: réaliser un dispositif fonctionnant en large bande spectrale jusqu'à $\lambda=60 \mathrm{~nm}$, avec un pouvoir résolvant de l'ordre de 500000 (FWHM) à la longueur d'onde minimale. Ces choix permettent de fixer les paramètres essentiels du balayage, y compris les tolérances.

Dans la pratique, l'interférogramme balayé en différence de marche est toujours échantillonné à intervalle régulier, ce qui permet l'utilisation de l'algorithme FFT. Cet intervalle doit être de l'ordre de $\delta_{\min }=30 \mathrm{~nm}$ au maximum, pour satisfaire le théorème d'échantillonnage à la plus courte longueur d'onde analysée, soit $\lambda=60 \mathrm{~nm}$. D'autre part, pour assurer la résolution $\mathrm{R}$ souhaitée, la différence de marche maximale doit être $\Delta_{\max }=\mathrm{R} * \delta_{\min }=15 \mathrm{~mm}$. Ceci correspond à l'enregistrement de $2 \mathrm{R}$ échantillons entre $-\Delta_{\max }$ et $+\Delta_{\max }$, si le spectre est obtenu par TF exponentielle de l'interférogramme, ou R échantillons entre 0 et $\Delta_{\max }$, si le spectre est obtenu par TF en cosinus (ce qui exploite les symétries intrinsèques $\mathrm{du}$ problème). Nous avons choisi cette deuxième option pour réduire la longueur du balayage et les difficultés associées. Les paramètres sont donc: nombre d'échantillons $512 \mathrm{~K}(1 \mathrm{~K}=1024)$, intervalle de différence de marche entre échantillons $\delta \approx 30 \mathrm{~nm}$, soit un balayage de 0 à $15 \mathrm{~mm}$ en ddm, $7.5 \mathrm{~mm}$ en déplacement du bras mobile (sect. 2.1).

La qualité et l'exactitude du spectre reposent sur la régularité du peigne d'échantillonnage. Sans entrer dans les détails, on peut montrer que toute erreur sur la position (en ddm) d'un échantillon se traduit par une déformation de la fonction d'appareil. Pour un interférogramme parfait, sans apodisation, 
celle-ci est un sinus cardinal. On admet que la déformation de la fonction d'appareil est admissible lorsque la hauteur du sinc n'est pas atténuée de plus de $10 \%$ (ce critère est de même nature que le critère de Maréchal en optique, qui définit la déformation admissible d'une surface d'onde). On montre que la condition nécessaire pour arriver à ce résultat est que les erreurs de position du peigne d'échantillonnage ne soient pas supérieures à $\lambda / 15$ en moyenne quadratique, $\lambda$ étant la longueur d'onde mesurée. Pour $\lambda=60 \mathrm{~nm}$, l'erreur tolérable sur la position des échantillons (en ddm) pour un spectre "parfait", est donc $4 \mathrm{~nm}$ toutes causes cumulées, en moyenne quadratique sur l'ensemble des échantillons.

\subsection{Stratégie pour un balayage exact}

On voit donc que, bien plus que l'interféromètre EUV proprement dit (statique), c'est la réalisation d'un système de balayage assurant la spécification ci-dessus qui constitue le défi principal. Globalement, deux approches sont possibles. Soit on réalise un système intrinsèquement précis, tel que le guidage et le déplacement du bras mobile satisfont par construction les spécifications imposées. C'est l'option retenue par Howells et al [4]. Nous avons considéré quant à nous qu'elle impliquait un effort technologique déraisonnable, et sans garantie de succès. Dans ces conditions, on doit nécessairement utiliser un montage dont les qualités intrinsèques sont insuffisantes, et lui adjoindre un système de mesure adhoc, capable de permettre d'une manière ou d'une autre la compensation des erreurs, de façon à assurer que l'échantillonnage a lieu aux valeurs de ddm requises. Par ailleurs, toujours en raison de la valeur très faible de l'intervalle entre échantillons, nous avons choisi de ne pas arrêter le déplacement sur les positions successives à échantillonner, mais d'échantillonner "au vol" l'interférogramme, le balayage ayant lieu à vitesse à peu près constante. Il reste à définir la stratégie de mesure, et à trouver une méthode pour la mettre en oeuvre.

Dans un interféromètre à balayage classique, on utilise un faisceau auxiliaire (laser stabilisé en fréquence par exemple) que l'on injecte dans l'interféromètre lui-même, par exemple à l'aide de lames séparatrices, de façon à mesurer directement la différence de marche, le chemin mesuré par le faisceau auxiliaire étant le même que celui vu par le faisceau principal. Dans le présent contexte, cette approche, sans être strictement impossible, pose un certain nombre de difficultés. En fait, la distance demandée entre échantillons, $15 \mathrm{~nm}$ en déplacement, semble peu compatible avec la valeur standard d'interfrange dans un interféromètre à deux ondes, $316 \mathrm{~nm}$ en déplacement avec la raie He-Ne. Toute opération conduisant à une interpolation importante (et précise) sur l'interfrange $\lambda / 2$ conduit nécessairement à une certaine complexité du dispositif auxiliaire, elle même peu compatible avec la structure choisie pour l'interféromètre EUV. Dans ces conditions, nous avons choisi de déporter la mesure et le contrôle du déplacement sur l'arrière de l'interféromètre EUV, selon le principe de la figure 3. L'optique comporte deux parties: le bras fixe, et le bras mobile, chacun étant constitué de deux éléments qui doivent être solidaires: le réflecteur EUV et une partie du système de contrôle. L'objectif est que le déplacement ainsi mesuré soit strictement proportionnel à la différence de marche EUV.

On peut d'abord remarquer que la mesure de déplacement effectuée est différentielle, c'est à dire qu'un mouvement de translation d'ensemble des deux bras de l'interféromètre ne sera pas pris en compte dans la mesure. Ceci relaxe de façon très importante la stabilité d'ensemble demandée au montage, dans la mesure ou tout mouvement d'ensemble ne peut perturber l'interférogramme enregistré qu'à l'échelle de l'interfrange (plus de $100 \mu \mathrm{m}$ ), au pire. Cette stabilité d'ensemble n'est donc absolument pas critique, et la mécanique de mise en place des réflecteurs peut être réalisée avec des techniques standards. Au contraire, les déplacements différentiels des blocs optiques, tels que mesurés "par l'arrière", doivent être représentatifs, à l'échelle de quelques nanomètres, des différences de marches réelles, du coté EUV. En fait, on peut voir que les erreurs différentielles proviennent essentiellement, soit des dilatations différentielles entre le bloc fixe et le bloc mobile, soit de rotations du bloc mobile pendant la translation (lacet, tangage). Pour maintenir les erreurs de ddm à un niveau tolérable, on doit agir sur deux paramètres: la minimisation des dilatations et des rotations parasites d'une part, et, en ce qui concerne les rotations, le choix du point de mesure d'autre part. 
Compte tenu de l'environnement d'un tel spectromètre, les dilatations différentielles sont certainement négligeables si les blocs optiques sont réalisés, en matériau à faible dilatation, tels que le Zérodur ${ }^{\circledR}$. Par ailleurs, l'analyse montre que l'influence du point de mesure sur les erreurs de ddm induites par les lacets et tangages parasites est critique, qu'elle dépend de la position (mal connue !) des axes de rotation parasites, et de la manière dont une éventuelle correction angulaire est appliquée. Au total, on trouve que le point de mesure du déplacement relatif doit être le plus proche possible du point d'incidence du faisceau EUV (ce qui n'est pas surprenant), et que, dans les conditions pratiques du montage proposé, les rotations parasites (tangage et lacet) doivent être maintenues inférieures à quelques dixièmes de microradians pour avoir une influence négligeable.

\section{DISPOSITIF DE CONTRÔLE}

\subsection{Principe de réalisation}

Le système de contrôle de déplacement est destiné à mesurer la différence de marche, selon les principes donnés au paragraphe précédent, en garantissant son exactitude. Il comporte donc un déflectomètre pour mesurer la déviation angulaire du réflecteur mobile, et une mesure du déplacement différentiel. Il est basé sur l'utilisation d'un laser He-Ne stabilisé en fréquence et monomode transverse (précision sur la fréquence quelques $10^{-8}$ sur plusieurs heures), qui traverse plusieurs fois l'intervalle entre les miroirs de contrôle des bras fixe et mobile. Ceci permet d'amplifier à la fois les rotations parasites du bloc mobile (en vue d'un asservissement sur une direction fixe), et ses déplacements (en vue d'une mesure interférométrique). De plus, pour définir de façon simple une direction intrinsèque au montage, le système est configuré pour que le faisceau de contrôle revienne rigoureusement sur lui même après un certain nombre de réflexions entre les miroirs. Ceci est réalisé grâce au schéma de la figure 4a. On montre facilement que la condition de "résonance" (retour du faisceau sur lui-même) est réalisée lorsque la première incidence sur le miroir mobile est $\mathrm{i}=(\mathrm{p}-1) \alpha$, ou $\alpha$ est l'angle entre les miroirs de contrôle, et $\mathrm{p}$ un entier, $2 \mathrm{p}-1$ étant le nombre total (aller-retour) de réflexions entre les miroirs. Nous utilisons $\mathrm{p}=11$, et $\alpha=1 \mathrm{deg}$. Pour des raisons pratiques, le faisceau du laser de contrôle est dédoublé en amont de l'interféromètre en deux faisceaux égaux, qui suivent deux trajets strictement parallèles entre les miroirs de contrôle, l'un pour la stabilisation angulaire, l'autre pour la mesure du déplacement.
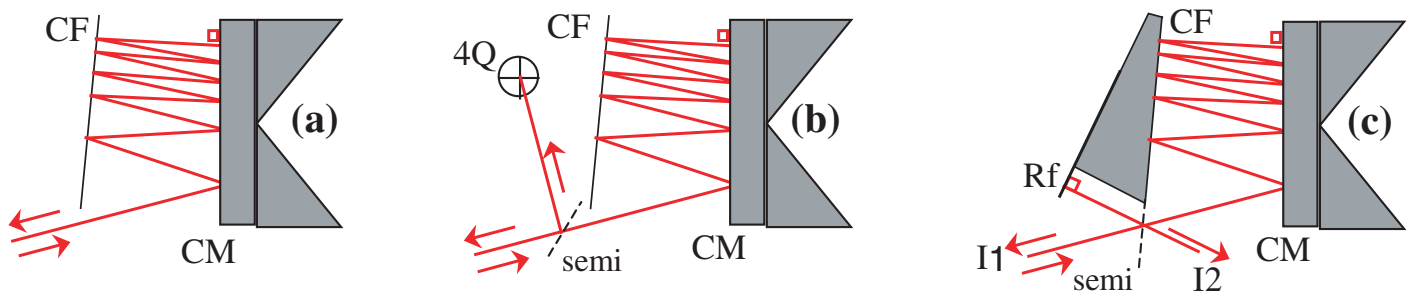

Figure 4. (a) Principe du syst'eme multipasse "résonant" (voir texte). (b) Utilisation en déflectométre. (c) Utilisation en interférométre à deux ondes (Michelson) à haute sensibilité. Le miroir de référence Rf fait partie du bloc optique du bras fixe. CF: miroir de contrôle du bloc fixe; CM: miroir de contrôle du bloc mobile; 4Q: cellule 4 quadrants. I1 et I2: sorties du Michelson de contrôle.

\subsection{Déflectomètre : contrôle de la direction du réflecteur mobile}

Pour mesurer les écarts angulaires au cours du déplacement, et asservir la direction du réflecteur pour une déviation minimale, on renvoie le faisceau retourné sur un détecteur 4-quadrants classique (fig 4b). Ce détecteur doit être réglé rigoureusement de façon à définir une position du faisceau retour confondue avec le faisceau incident. L'intervalle minimum entre les miroirs de contrôle étant de $15 \mathrm{~mm}$ minimum, et la cellule étant placée à $200 \mathrm{~mm}$ de la première réflexion, on trouve que le déplacement linéaire du 
faisceau retourné sur la 4-quadrants est au moins de $7.7 \mu \mathrm{m} / \mu \mathrm{rad}$. Par ailleurs, on trouve que le bruit mesuré sur le détecteur en l'absence de déplacement du miroir mobile est équivalent à un déplacement de l'ordre de $1 \mu \mathrm{m}$. On en déduit que le système peut stabiliser la position angulaire au cours du déplacement avec une erreur inférieure à $0.15 \mu \mathrm{rad}$.

\subsection{Interféromètre : mesure de la position du réflecteur mobile}

Pour mesurer le déplacement relatif des miroirs de contrôle, on utilise le trajet multipassage entre ces deux miroirs comme l'un des bras d'un interféromètre de Michelson, selon le schéma de la figure 4c (déjà été utilisé dans [5]). Bien entendu, les bras sont très loin d'être équilibrés puisque le bras mobile développé peut être aussi long que $500 \mathrm{~mm}$, pour 10 à $20 \mathrm{~mm}$ pour le bras de référence. Ce déséquilibre est permis par l'utilisation du laser stabilisé en fréquence, qui garantit mieux que $10^{-8}$ sur 8 heures. Mais il impose une stabilité équivalente pour le bras de référence. Celui-ci est donc défini par une cale en Zérodur, dont le coefficient de dilatation thermique est inférieur à $10^{-7} /{ }^{\circ} \mathrm{K}$ à la température ordinaire. Dans ces conditions, on voit que l'interféromètre de contrôle décrit une frange lorsque le miroir mobile se déplace d'environ $\lambda / 22=28.76 \mathrm{~nm}$. Un calcul exact tenant compte des incidences non normales donne $28.94 \mathrm{~nm}$. Il est facile de fabriquer à partir de ce signal un peigne d'impulsions calé sur la demi-frange, soit tous les $28.94 \mathrm{~nm}$ en différence de marche, ce qui était le but recherché.

\section{TESTS ET PREMIÈRES MESURES}

Le dispositif que nous venons de décrire à d'abord été construit sur banc, dans l'environnement d'un laboratoire d'optique, mais avec le dimensionnement nécessaire au fonctionnement nominal. Seule la mise sous vide n'a pas été intégrée à cette étape. Dans un premier temps, nous avons mesuré des spectres visibles et proche UV pour vérifier le fonctionnement du système et la validité des concepts mis en œuvre. Par la suite nous avons procédé à l'intégration de l'ensemble du système (optique, commandes et mesures, à l'exception notable du laser de contrôle) dans une enceinte à vide, en vue de sa connection à l'anneau synchrotron SuperACO du LURE (Orsay, ligne SU5).

\subsection{Mesure des erreurs du peigne d'échantillonnage et correction des erreurs systématiques}

La première expérience a consisté à mesurer le spectre du laser HeNe de contrôle, en injectant une fraction du faisceau du coté EUV. La largeur de raie laser étant très inférieure à la résolution spectrale du système, on obtient directement la réponse percussionnelle du spectromètre, qui a été trouvée très proche de sa forme théorique, un sinus cardinal. Toutefois, il ne faut pas oublier que l'influence des erreurs de position de l'échantillonnage est inversement proportionnelle à la longueur d'onde analysée. En d'autres termes, un niveau d'erreur juste acceptable à $600 \mathrm{~nm}$ donne une réponse fortement dégradée à $60 \mathrm{~nm}$. Il n'est donc pas sans intérêt de chercher à évaluer plus précisément ces erreurs de position.

Or l'exploitation du même interférogramme permet d'obtenir le profil des erreurs d'échantillonnage en fonction de la différence de marche. Ceci résulte du fait que l'interférogramme idéal du laser monofréquence étant une sinusoïde, tout écart au spectre idéal (sinc) résulte d'une perturbation du processus d'acquisition de l'interférogramme. En particulier, des décalages du peigne d'échantillonnage équivalent à des erreurs de phase d'une porteuse sinusoïdale. On peut donc réaliser une opération classique de démodulation en phase d'une porteuse, pour extraire de l'interférogramme un profil lissé des erreurs d'échantillonnage en fonction de la différence de marche. La fenêtre de lissage est typiquement large de 2000 à 8000 échantillons. Des tests systématiques ont montré que le système (déplacement + contrôle) introduit dans la mesure une erreur d'échantillonnage qui peut être aussi faible que $6 \mathrm{~nm} \mathrm{rms}$, lorsque le système est bien réglé. Ce niveau d'erreur correspond au niveau admissible à $\lambda=90 \mathrm{~nm}$, pour une réponse "parfaite". De plus, on constate que, pour un réglage donné de l'ensemble des faisceaux (EUV 
et contrôle), le profil d'erreur est largement systématique, la part non systématique étant inférieure à la limite des $4 \mathrm{~nm}$ rms.

Il est donc possible, si nécessaire, de mettre en place une procédure systématique de correction fine des erreurs d'échantillonnage (au moins de la part basse fréquence du profil d'erreur). Il suffit pour cela d'injecter un faisceau auxiliaire dans l'interféromètre coté EUV, et d'enregistrer simultanément le signal d'interférence EUV et le signal provenant du faisceau de référence. On peut alors extraire le profil d'erreur en filtrant fortement la raie de référence. Ce profil étant basse fréquence, il est localement constant et on peut procéder à une correction de l'interférogramme, par une interpolation classique en sinc [3], de valeur localement constante. Cette procédure a été testée sur la raie du laser de référence, et a montré un excellent niveau de correction, même lorsqu'on utilise un profil d'erreur moyen, déduit d'un ensemble de mesures systématiques préalables.

Il faut également noter qu'un tel interférogramme de référence fournirait la possibilité d'un autoétalonnage de l'échelle spectrale.

\subsection{Mesure du spectre de l'oxygène dans le proche UV $(\lambda=200-185 \mathrm{~nm})$}

Après intégration de l'interféromètre dans son enceinte à vide, le système a été mis en place sur l'anneau SuperACO de LURE (Orsay), ligne onduleur SU5. Dans un premier temps nous avons testé l'ensemble du système, dans l'ambiance de l'anneau superACO (vibrations de dalle, bruits acoustiques, bruits électroniques), mais à l'air. L'onduleur a été réglé pour rayonner vers $200 \mathrm{~nm}$, région comportant une série bien connue de bandes d'absorption de l'oxygène (bandes de Schumann-Runge), qui ont par ailleurs été étudiées de façon très détaillée et tabulées [6, 7]. L'épaisseur d'air traversée était de $150 \mathrm{~cm}$ environ. Les acquisitions d'interférogrammes comportaient $540 \mathrm{~K}$ échantillons environ, pour assurer la présence de la frange de ddm nulle, et permettre la détermination précise de sa position par la procédure classique du recalage de phase sur un interférogramme symétrique à "faible" différence de marche $(+/-16 \mathrm{~K}$ échantillons soit une $\mathrm{ddm} \mathrm{de}+/-0.25 \mathrm{~mm})$. La figure 5 a montre le spectre calculé à partir d'un interférogramme typique, entre $\lambda=202 \mathrm{~nm}$ et $\lambda=185 \mathrm{~nm}$ soit 12000 éléments spectraux séparés
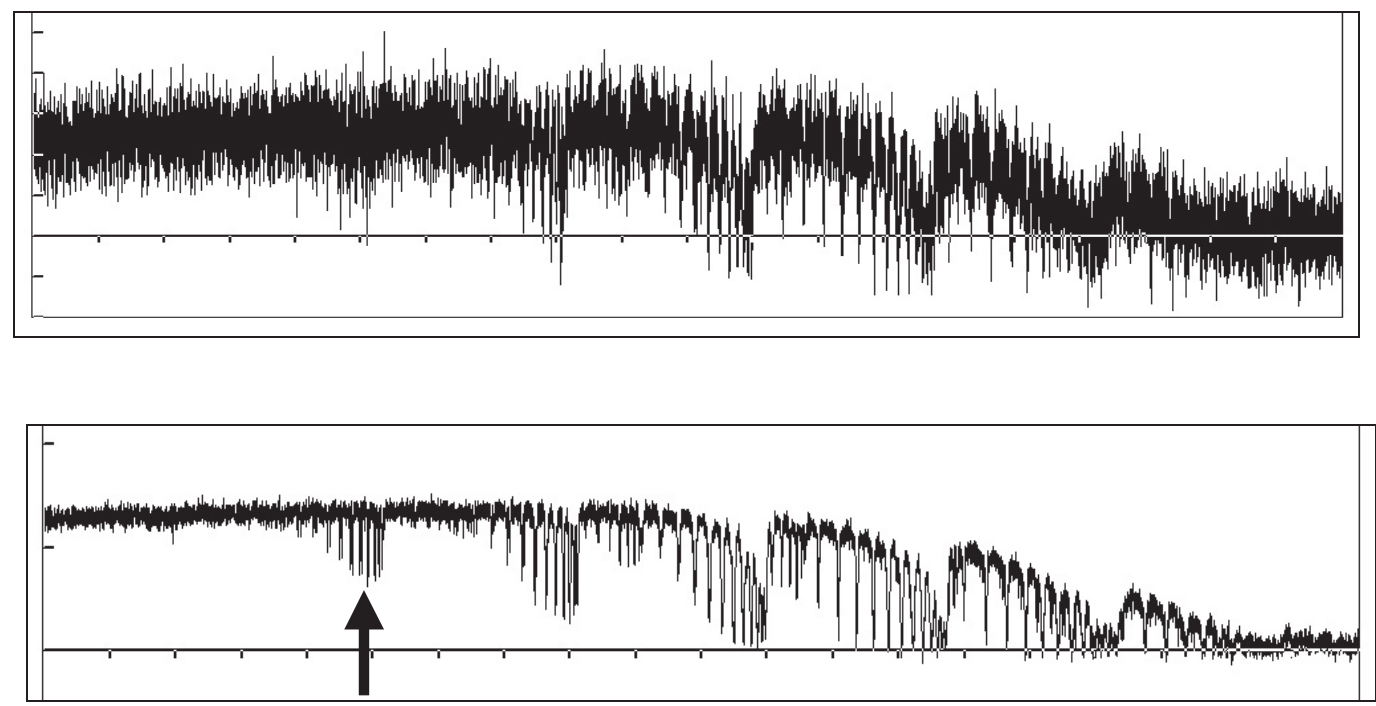

Figure 5. (a) en haut, spectre calculé à partir d'un seul interférogramme; (b) moyenne de 50 spectres obtenus dans les mêmes conditions. (a) et (b): largeur spectrale vue: 12000 échantillons spectraux espacés de $0.36 \mathrm{~cm}^{-1}$ soit de 49600 à $54000 \mathrm{~cm}^{-1}(202$ à $185 \mathrm{~nm})$. Les bandes d'absorption sont les bandes de Schuman-Runge de $\mathrm{O}_{2}$. La flèche indique la région agrandie sur la figure 6. 
de $0.36 \mathrm{~cm}^{-1}$. On voit la bosse due à l'émission de l'onduleur vers $200 \mathrm{~nm}$, dans laquelle s'inscrivent des structures spectrales. Le calcul montre que le bruit très important provient entièrement du bruit de photon du faisceau analysé. On peut donc améliorer le rapport $\mathrm{s} / \mathrm{b}$ d'un facteur $\sqrt{N}$ en enregistrant successivement $\mathrm{N}$ interférogrammes et en additionnant les spectres obtenus. Nous avons appliqué cette technique, classique en spectrométrie par $\mathrm{TF}$, avec $\mathrm{N}=50$. La figure $5 \mathrm{~b}$ montre le résultat, avec la même échelle spectrale que la figure 5a. Sur la figure 6, l'échelle spectrale a été dilatée (400 éléments spectraux) pour montrer la résolution réelle obtenu. Outre le spectre moyen mesuré, cette figure montre la courbe d'absorption calculée, dans nos conditions expérimentales, à partir des données publiées [7]. L'accord est excellent dans l'ensemble de la bande spectrale.

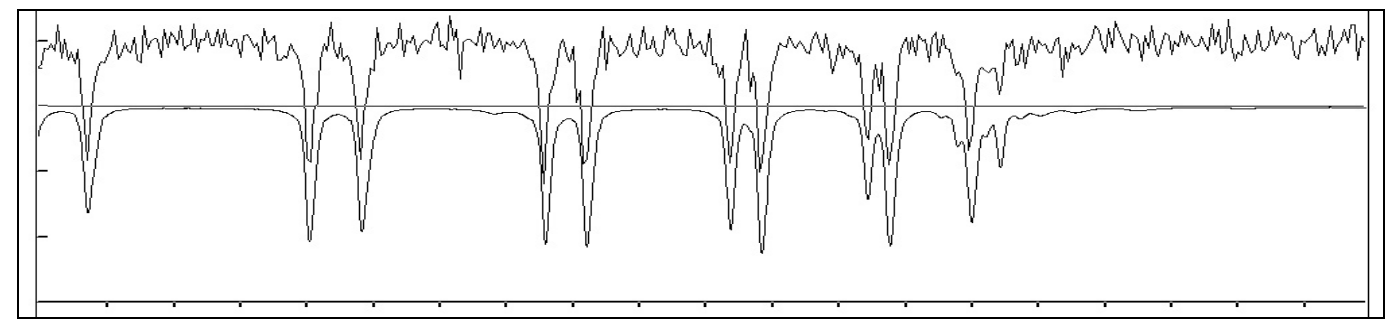

Figure 6. La trace supérieure est un agrandissement de la figure 5b, montrant 400 échantillons spectraux de 50608 à $50752 \mathrm{~cm}^{-1}$ environ. La trace inférieure est la courbe de transmission issue des table ref [7], calculée pour nos conditions expérimentales. Elle est décalée vers le bas pour faciliter la lecture. La ligne horizontale correspond à la transmission 1.

\section{CONCLUSION}

L'obstacle majeur à une large extension de la spectrométrie par TF dans le domaine EUV (voire XUV) peut être contourné par l'utilisation d'un interféromètre à division de front d'onde. Nous avons démontré qu'il est possible de concevoir et réaliser un interféromètre à balayage de ce type, en vue d'une spectrométrie par TF dans le domaine 60-150 nm, avec des performances inconnues dans ce domaine spectral. Les premières mesures, autour de $\lambda=195 \mathrm{~nm}$, démontrent un fonctionnement nominal. Nous pensons que la résolution devrait, à terme, excéder largement les spécifications prévues $(\mathrm{R}=6$ à 800000 à $80 \mathrm{~nm}$, au lieu de 400 000). L'instrument doit être installé au cours de l'année 2006 sur la ligne DESIRS de la source synchrotron SOLEIL, en cours de construction.

\section{Bibliographie}

[1] D. Joyeux, F. Polack and D. Phalippou, Rev. Sci. Intrum. 70, 7 (1999)

[2] L. Nahon, C. Alcaraz, J.L. Marlats, B. Lagarde, F. Polack, R. Thissen., D. Lepere, K. Ito, Rev. Sci. Instrum 72, 1320 (2001)

[3] J. Chamberlain, The principles of interferometric spectroscopy, (J. Wiley, New York, 1979)

[4] M.R. Howells, K. Frank, Z. Hussain, E.J. Moler, T. Reich, D. Moller, D.A. Shirley, Nucl. Instr. and Methods in Physics Research 347, 182 (1994)

[5] S. Chandra and R.S. Rohde, App. Opt. 21, 1533 (1982)

[6] K. Yoshino, D.E. Freeman, J.R. Esmond, W.H. Parkinson, Planetary Space Science, 31, 3 (1983) K. Yoshino, J.R. Esmond, A.S.-C. Cheung, D.E. Freeman, W.H. Parkinson, Planetary Space Science, 40, 2/3, (1992)

[7] Voir site web: http://cfa-www.harvard.edu/amdata/ampdata/o2pub92/S-R.html 